\title{
Reply to "Association Between Concomitant Mediastinoscopy and Postoperative Pneumonia After Pulmonary Lobectomy"
}

\author{
Sai Yendamuri, MD, FACS ${ }^{1}$ and Kris Attwood, PhD $^{2}$ \\ ${ }^{1}$ Department of Thoracic Surgery, Roswell Park Cancer Institute, Buffalo, NY; ${ }^{2}$ Department of Biostatistics, Roswell Park \\ Cancer Institute, Buffalo, NY
}

We thank Drs. Yu, Li, and Yang for their comments on our recent publication. ${ }^{1}$ Although already stated in the manuscript, we would like to reiterate that we do recognize that the comparison groups have differences between them, hence multivariable analyses have been performed and presented. The additional benefit of a propensity scorematched analysis is questionable. Several authors found no difference between multivariable and propensity-adjusted effect estimates. $^{2}$

However, for confirmation of the multivariable results, the effect of concomitant mediastinoscopy on the risk of postoperative pneumonia was estimated using propensity score methods to adjust for baseline covariate imbalances between the two cohorts. These methods offer another approach to combat confounding by measured covariates. The propensity score (PS) was estimated with a multivariable logistic regression model for the probability of concomitant mediastinoscopy. Covariates included in the model depended on the dataset and are found in Tables 1 and 4 of the original article. ${ }^{1}$ Based on the approach outlined by Stürmer et al., ${ }^{3}$ the propensity scores were then trimmed and inverse probability weights (IPW) were calculated. A weighted logistic regression model, weighted by the IPW, was then used to estimate the effect of concomitant mediastinoscopy on the risk of postoperative pneumonia.

Table 1 shows that the results are relatively consistent with both the original univariate and multivariable analyses and demonstrate a significant impact of concomitant

\section{(C) Society of Surgical Oncology 2018}

First Received: 28 March 2018;

Published Online: 18 April 2018

S. Yendamuri, MD, FACS

e-mail: sai.yendamuri@ roswellpark.org
TABLE 1 Results of the association of concomitant mediastinoscopy with postoperative pneumonia using propensity score-matched analysis

\begin{tabular}{llll}
\hline Dataset & Sample & Adjusted odds ratio $(95 \% \mathrm{CI})$ & $P$ value \\
\hline RPCI & Overall & $1.46(0.99-2.12)$ & 0.052 \\
& Open & $0.66(0.26-1.64)$ & 0.37 \\
& VATS & $1.74(1.13-2.66)$ & 0.011 \\
\multirow{2}{*}{ NSQIP } & Overall & $1.50(1.36-1.64)$ & $<0.001$ \\
& Open & $1.52(1.36-1.71)$ & $<0.001$ \\
& VATS & $1.47(1.25-1.74)$ & $<0.001$ \\
\hline
\end{tabular}

Higher odds ratio shows an association with increased postoperative pneumonia

RPCI Roswell Park Cancer Institute cohort, NSQIP National Surgical Quality Improvement Program, CI confidence interval, VATS videoassisted thoracoscopic surgery

mediastinoscopy on the risk of postoperative pneumonia. We emphasize again that this does not eliminate bias from the retrospective nature of the study. The results we see need to be examined in other datasets and in prospective studies.

\section{REFERENCES}

1. Yendamuri S, Battoo A, Attwood K, Dhillon SS, Dy GK, Hennon M, Picone A, Nwogu C, Demmy T, Dexter E. Concomitant mediastinoscopy increases the risk of postoperative pneumonia after pulmonary lobectomy. Ann Surg Oncol. 2018. https://doi.org/ 10.1245/s10434-018-6397-6.

2. Shah BR, Laupacis A, Hux JE, Austin PC. Propensity score methods gave similar results to traditional regression modeling in observational studies: a systematic review. J Clin Epidemiol. 2005;58(6):550-9. https://doi.org/10.1016/j.jclinepi.2004.10.016.

3. Sturmer T, Wyss R, Glynn RJ, Brookhart MA. Propensity scores for confounder adjustment when assessing the effects of medical interventions using nonexperimental study designs. J Intern Med. 2014;275(6):570-80. https://doi.org/10.1111/joim.12197. 